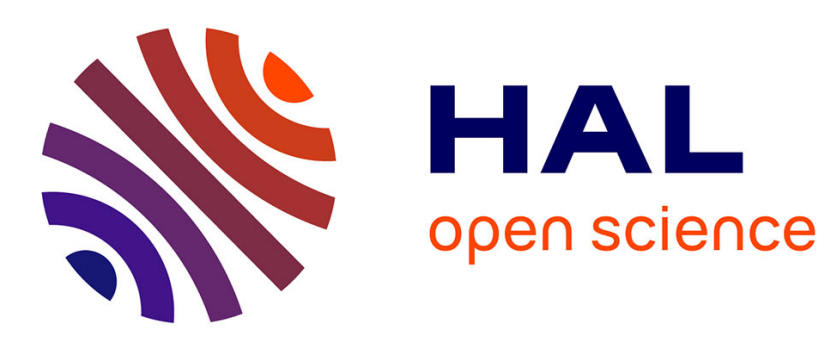

\title{
Oblique Mixed Shocks in Extragalactic Jets
}

Didier Fraix-Burnet, Guy Pelletier

\section{- To cite this version:}

Didier Fraix-Burnet, Guy Pelletier. Oblique Mixed Shocks in Extragalactic Jets. The Astrophysical Journal, 1991, 367, pp.86-95. hal-00002330

\section{HAL Id: hal-00002330 \\ https://hal.science/hal-00002330}

Submitted on 27 Jul 2004

HAL is a multi-disciplinary open access archive for the deposit and dissemination of scientific research documents, whether they are published or not. The documents may come from teaching and research institutions in France or abroad, or from public or private research centers.
L'archive ouverte pluridisciplinaire HAL, est destinée au dépôt et à la diffusion de documents scientifiques de niveau recherche, publiés ou non, émanant des établissements d'enseignement et de recherche français ou étrangers, des laboratoires publics ou privés. 


\title{
OBLIQUE MIXED SHOCKS IN EXTRAGALACTIC JETS
}

\author{
D. FRAIX-BURNET* \\ Observatoire Midi-Pyrénées URA 285, France \\ AND \\ G. PELLETIER \\ Observatoire de Grenoble URA 708, France
}

Received: April 26, 1990; Accepted: July 11, 1990

To appear in the January 20, 1991 issue of The Astrophysical Journal

* Now at the Space Telescope Science Institute, 3700 San Martin Drive, Baltimore, MD 21218, USA. Affiliated to the Astrophysics Division, Space Science Department, European Space Agency. 


\begin{abstract}
Analytical calculations of the spectra obtained from first-order Fermi acceleration mechanism with oblique shocks are presented. They are a generalization of the quasiparallel configuration, where the streaming instability modifies the acceleration process, and of the quasiperpendicular configuration, where the magnetic pressure is significant. We show that influence of the streaming instability on the compression ratio of the fluid is always negligible, it changes the spectal index only through the modification of the effective compression ratio of the scattering centers. The observational constraints on the cut-off frequency imply that the magnetic turbulence in extragalactic jets must be weak and most probably of Kraichnan type. The application of our calculations to the jet of $3 \mathrm{C} 273$ shows that the theory of diffusive acceleration in oblique shocks works quite well with a non-relativistic flow velocity, and yields narrow ranges for the different parameters.
\end{abstract}

Subject headings: galaxies: jets - particle acceleration - shock waves 


\section{Introduction}

Particle acceleration in astrophysics has been proved to be of extreme importance for cosmic rays and extragalactic radio sources. The concept of diffusive shock acceleration, invented twelve years ago by several authors (Axford et al. 1977, Krimsky 1977, Bell 1978 and Blandford and Ostriker 1978), has been widely used to explain the spectra of high energy particles in supernovae remnants and radiosources. Although the simple linear theory firstly proposed needs some extensions to become a realistic description, the basic mechanism is still considered as the relevant process at work that accelerates efficiently high energy particles.

Most of the published works deal with simplest configurations, namely parallel or quasiparallel and quasiperpendicular shocks; the theory of particle acceleration in perpendicular shocks is different because the electric field cannot be removed by a de Hoffmann-Teller transformation (Begelman and Kirk 1990). Unfortunately, the calculations are more complex for oblique shocks, and thus it is more difficult to know the relative importance of different parameters due to the lack of any analytical theory. Oblique shocks have been considered only in recent years (Drury 1983, Webb 1983, Ostrowski 1988, Kirk and Heavens 1989, Cawthorne and Cobb 1990). However, they probably play an important role to make the bright jets shine (Norman et al. 1982).

In previous papers, Pelletier and Roland (1986, hereafter Paper 1; 1988, hereafter Paper 2) developed analytical calculations of the spectra obtained from the first-order Fermi acceleration mechanism at work in magnetized mixed shocks for both quasiparallel and quasiperpendicular configurations. The plasma is supposed to have two distinct components, namely a nonrelativistic thermal one and an ultrarelativistic suprathermal one. The upstream flow is assumed to have a nonrelativistic velocity. Analitycal results can be obtained because the shock structure can be simply modeled owing to the great differences between the three different scales involved in the problem: the synchrotron loss length, the precursor length which is the typical scale of the diffusion of relativistic electrons, and finally the width of the subshock, which is the shock of the thermal component. Pelletier and Roland calculated the spectral index as a function of $\theta$ (ratio between the thermal pressure and the relativistic plasma pressure, defined just downstream of the shock where synchrotron losses are still not significant) first for infinite Alfven Mach number $\left(M_{A}\right)$ in Paper 1, then for finite $M_{A}$ in Paper 2 taking account of the streaming instability.

In this Paper, we generalize these calculations to oblique shocks and follow very closely the methods of the two previous Papers. We take into account both streaming instability and magnetic pressure effects. The shapes of the precursor and of the subshock are described at the lowest order in term of the small number $M_{A}^{-1}$. The configuration of the shock is defined in the reference frame where the shock front is at rest and the upstream speed of the thermal plasma is perpendicular to it. The obliquity is then defined by the angle $\beta^{u}$ between the magnetic field and the normal to the shock front, or the upstream plasma speed (Fig. 1).

\section{The derivation of the spectral index}




\subsection{The continuity of $\vec{v}$ and $\vec{B}$}

First we have to address the behavior of the flow speed $(\vec{v})$ and the magnetic field $(\vec{B})$ across the shock. The normal to the shock front defines the $x$-axis, and the vectors $\vec{v}$ and $\vec{B}$ are in the plane $x-y$. The equation for the conservation of momentum reads:

$$
\left\{\begin{array}{l}
\rho v_{x} \frac{\partial v_{x}}{\partial x}=-\frac{\partial p}{\partial x}-\frac{1}{4 \pi} B_{y} \frac{\partial B_{y}}{\partial x} \\
\rho v_{x} \frac{\partial v_{y}}{\partial x}=\frac{1}{4 \pi} B_{x} \frac{\partial B_{y}}{\partial x}
\end{array} .\right.
$$

The conservation of mass implies that $\rho v_{x}=J$ where $J$ is a constant, and the equations (1) can be integrated into:

$$
\left\{\begin{array}{l}
J v_{x}+p+p_{m}=C s t \\
J v_{y}-\frac{B_{x} B_{y}}{4 \pi}=C s t
\end{array}\right.
$$

where $p_{m}=\frac{B_{y}^{2}}{8 \pi}$ is the magnetic pressure and $\frac{B_{x} B_{y}}{4 \pi}$ the magnetic stress. $B_{x}$ is the component of the magnetic field normal to the shock front $\left(B_{x}^{u}=B_{x}^{d}=B_{x}\right.$ : the upperscripts $u$ and $d$ mean respectively upstream and downstream quantities). With the conservation of the electric field parallel to the shock front $(\vec{E}=-\vec{v} \wedge \vec{B})$, equation (2) yields:

$$
\begin{gathered}
\left\{\begin{array}{l}
B_{y}^{d}=r s B_{y}^{u} \\
v_{y}^{d}=(s-1) \frac{B_{y}^{u}}{B_{x}} v^{u}
\end{array}\right. \\
\text { with } \quad s=\left(\frac{1-\frac{B_{x}^{2}}{4 \pi J v^{u}}}{1-r \frac{B_{x}^{2}}{4 \pi J v^{u}}}\right)
\end{gathered}
$$

where $\mathrm{r}$ is the global compression ratio $r=v_{x}^{u} / v_{x}^{d}=v^{u} / v_{x}^{d}$.

We then see that:

$$
p_{m}^{d}=r^{2} p_{m}^{u} s^{2}
$$

Defining $M_{A}$ as the Alfvenic Mach number upstream $M_{A}=v^{u} / V_{A}$, where $V_{A}^{2}=\frac{B^{u 2}}{4 \pi \rho^{u}}$, we have:

$$
\frac{B_{x}^{2}}{4 \pi J v^{u}}=\frac{\cos ^{2} \beta^{u} B^{u 2}}{4 \pi \rho^{u} v^{u 2}}=\frac{\cos ^{2} \beta^{u}}{M_{A}^{2}},
$$

and $s=\left(M_{A}^{2}-\cos ^{2} \beta^{u}\right) /\left(M_{A}^{2}-r \cos ^{2} \beta^{u}\right)$.

This means that the magnetic stress induces an effect of the second order in $M_{A}^{-1}$. Hence, to the first order $\left(M_{A} \gg 1\right)$, we find: $s=1+\mathrm{o}\left(M_{A}^{-1}\right)$, so that we can write:

$$
\left\{\begin{aligned}
B_{y}^{d} & =r B_{y}^{u} \\
v_{y}^{d} & =v_{y}^{u}=0 \\
p_{m}^{d} & =r^{2} p_{m}^{u}
\end{aligned}\right.
$$


which is the result of the parallel and perpendicular cases where the magnetic stress vanishes. In the following, we consider only the $x$-component of the speed downstream, thus omitting the corresponding subscript $\left(v_{x}^{d}=v^{d}\right)$, but we limit the calculation to the first order in $M_{A}^{-1}$ only at the end (Sect. 2.4).

\subsection{The basic equations}

The streaming instability induces upstream a difference between the speeds of the thermal plasma $\left(v^{u}\right)$ and the scattering centers $\left(u^{u}\right.$; Skilling 1975, Mc Kenzie and Volk 1982):

$$
u^{u}=v^{u}-V_{A} \cos \beta^{u}
$$

We assume that the streaming instability drives an intense spectrum of Alfven waves that propagate backwards in the precursor and that the power supply to the waves by the instability is balanced, via a transfer, by some absorption mechanism that heats the thermal plasma at a rate $V_{A} \frac{\partial p_{r}}{\partial x}$ (Völk, Drury, McKenzie, 1984).

The basic equations, which express respectively the momentum flux conservation, the thermal component heating and the relativistic pressure growth, are thus (see Paper 1):

$$
\left\{\begin{aligned}
J(u+V)+p_{g}+p_{r}+p_{m} & =F \\
\gamma_{g} p_{g} \frac{d(u+V)}{d u}+(u+V) \frac{d p_{g}}{d u} & =\delta \frac{d}{d u} \phi \frac{d p_{g}}{d u}+\left(\gamma_{g}-1\right) V \frac{d p_{r}}{d u} \\
\gamma_{r} p_{r}+u \frac{d p_{r}}{d u} & =\frac{d}{d u} \phi \frac{d p_{r}}{d u}
\end{aligned}\right.
$$

where $V=V_{A} \cos \beta^{u}$ upstream and $V=0$ downstream. The structure function $\phi$ was the key to solve this system of equations in Paper 1 and Paper 2 and is defined by $\phi(u)=\kappa_{r} \frac{\partial u}{\partial x}$ where $\kappa_{r}$ is the diffusivity of the relativistic particles. In equation (8), $p_{g}$ and $p_{r}$ are respectively the pressures of the thermal plasma $\left(\gamma_{g}=5 / 3\right)$ and of the relativistic particles for which the adiabatic index will be kept constant throughout the shock region $\left(\gamma_{r}=4 / 3\right)$.

\subsection{Relation between $b$ and $M_{A}$ in the strong shock limit}

We define $b$ as in Paper 2 by: $b=\left(p_{g}^{d}+p_{r}^{d}\right) / p_{m}^{d}$. In the strong shock limit $\left(p_{g}^{u}+p_{r}^{u} \ll J v^{u}\right)$, the momentum conservation reads:

$$
J v^{u}+p_{m}^{u}=p_{r}^{d}+p_{g}^{d}+p_{m}^{d}+J v^{d}
$$

which implies using (4):

$$
M_{A}^{2} \simeq \frac{r^{3} \sin ^{2} \beta^{u}}{2(r-1)}(b+1) s^{2}
$$

\subsection{Derivation of the compression ratio}

We use the same method as described in Appendix A of Paper 2, but we take the magnetic pressure $p_{m}$ into account: 


$$
\begin{gathered}
d\left(p_{g}+p_{r}+p_{m}\right)+J d v=0 \\
\int_{v^{d}}^{v^{u}}\left(\gamma_{g} p_{g} d v+v d p_{g}\right)=\left(\gamma_{g}-1\right) \int_{v^{d}}^{v^{u}} V d p_{r} \\
\int_{v^{d}}^{v^{u}}\left(\gamma_{r} p_{r} d u+v d p_{r}\right)=\int_{v^{d}}^{v^{u}} V d p_{r}
\end{gathered}
$$

(Note that there is an error in Appendix A of Paper 2 in the third equation where the term $\gamma_{r} p_{r} d u$ had been replaced by $\gamma_{r} p_{r} d v$, yielding an overestimation of the streaming instability.)

From equations (12) and (13), we obtain:

$$
\left[\left(\frac{\gamma_{g} p_{g}}{\gamma_{g}-1}+\frac{\gamma_{r} p_{r}}{\gamma_{r}-1}\right) v\right]_{v^{d}}^{v^{u}}-\int_{v^{d}}^{v^{u}} v\left(d p_{g}+d p_{r}\right)=\frac{\gamma_{r}}{\gamma_{r}-1} p_{r}^{u} V_{A} \cos \beta^{u} .
$$

Defining $\mu$ in the same way as in Paper 2:

$$
\mu=\frac{\frac{\gamma_{g}}{\gamma_{g}-1} p_{g}+\frac{\gamma_{r}}{\gamma_{r}-1} p_{r}+2 p_{m}}{p}
$$

with $p=p_{g}+p_{r}+p_{m}$, we derive:

$$
\frac{J\left(v^{u 2}-v^{d 2}\right)}{2}+\mu^{u} p^{u} v^{u}-\mu^{d} p^{d} v^{d}-2\left(p_{m}^{u} v^{u}-p_{m}^{d} v^{d}\right)+\int_{v^{d}}^{v^{u}} v d p_{m}=\frac{\gamma_{r}}{\gamma_{r}-1} p_{r}^{u} V_{A} \cos \beta^{u}
$$

There are two additive terms which are functions of the magnetic pressure. For the first term we have:

$$
-2\left(p_{m}^{u} v^{u}-p_{m}^{d} v^{d}\right)=-2 p_{m}^{u}\left(v^{u}-r^{2} s^{2} v^{d}\right) \quad .
$$

For the second term, equation (4) yields a relation across the shock between the magnetic pressure $p_{m}$ and the thermal plasma speed $v$ :

$$
p_{m}=p_{m}^{u}\left(\frac{v^{u}-\frac{B_{x}^{2}}{4 \pi J}}{v-\frac{B_{x}^{2}}{4 \pi J}}\right)^{2}
$$

Hence, we can integrate $v d p_{m}$ by parts with $v$ as variable to find:

$$
\int_{v^{d}}^{v^{u}} v d p_{m}=2 p_{m}^{u}\left(v^{u}-r^{2} s^{2} v^{d}-\frac{B_{x}^{2}}{8 \pi J}\left(1-r^{2} s^{2}\right)\right),
$$

and the sum of equations (17) and (19) yields:

$$
-p_{m}^{u} \frac{B_{x}^{2}}{4 \pi J}\left(1-r^{2} s^{2}\right)
$$


Equation (16) can then be transformed into:

$\frac{2 \mu^{d}-1}{r}=1+\frac{r \mu^{u}-\mu^{d}}{r-1} \frac{2 p^{u}}{J v^{u}}-\frac{2 r}{r-1} \frac{\gamma_{r}}{\gamma_{r}-1} \frac{p_{r}^{u} V_{A} \cos \beta^{u}}{J v^{u 2}}-2 \frac{r}{r-1} \frac{p_{m}^{u}}{J v^{u}} \frac{B_{x}^{2}}{4 \pi J v^{u}}\left(1-r^{2} s^{2}\right)$.

In the strong shock limit, we have $p^{u} \simeq p_{m}^{u}$, so that:

$$
\frac{2 p^{u}}{J v^{u}} \simeq \frac{2 p_{m}^{u}}{J v^{u}}=\frac{\sin ^{2} \beta^{u}}{M_{A}^{2}}
$$

and

$$
\mu^{u} \simeq 2
$$

The last term of the right hand side of equation (21) is proportional to $M_{A}^{-4}$ (see Sect. 2.1) and

$$
\frac{p_{r}^{u} V_{A}}{J v^{u 2}}<\frac{p^{u}}{J v^{u}} \frac{V_{A}}{v^{u}} \simeq \frac{\sin ^{2} \beta^{u}}{2 M_{A}^{2}} \frac{1}{M_{A}}
$$

so that the third term on the right hand side of equation (21) is at most of the third order in $M_{A}^{-1}$. This means that the streaming instability has a negligible effect on the global compression ratio of the thermal plasma.

Like in Paper 2, we can calculate $\mu^{d}$ by using

$$
r_{0}=\frac{7+4 \theta}{1+\theta}
$$

where $\theta=p_{g} / p_{r}$, together with equations (10) and (15). Then, if we neglect terms that are $\mathrm{o}\left(M_{A}^{-2}\right)$, equation $(21)$ becomes:

$$
r_{0}-\frac{r_{0}-3}{2} \frac{r^{3}}{r-1} \frac{\sin ^{2} \beta^{u}}{M_{A}^{2}}=r\left(1+\frac{4 r-r_{0}-1}{r-1} \frac{\sin ^{2} \beta^{u}}{2 M_{A}^{2}}\right),
$$

that can be rewritten as:

$$
r=\frac{r_{0}}{1+\frac{r\left(r_{0}-3\right)+r_{0}+1}{2} \frac{\sin ^{2} \beta^{u}}{M_{A}^{2}}} .
$$

The compression ratio $r$ as a function of the obliquity $\beta^{u}$ is plotted in Fig. 2 for different values of $\theta$ and $M_{A}$. It can be seen that the effect of the magnetic pressure on the compression ratio is visible at obliquities as low as $10^{\circ}$ and is important at high obliquities for moderate $M_{A}$.

\subsection{Derivation of the spectral index}

Like in Paper 2, we obtain a simple law for the low frequency radio spectral index valid for a wide parameter range provided that there is enough thermal pressure, precisely more 
than only a tenth of the relativistic pressure. Indeed the energy spectral index is related to $r$ in the case $\theta>10^{-1}$ by:

$$
\eta=\frac{3 r_{s}}{r_{s}-1}-2
$$

where $r_{s}$ is the compression ratio experienced by the scattering centers which, according to equation (7), reads

$$
r_{s}=r\left(1-\frac{\cos \beta^{u}}{M_{A}}\right)
$$

Equation (28b) provides the main influence of the streaming instability on the spectral index. In that range, the energy spectral index depends on the main control parameters of the shock structure and is not sensitive to the diffusion coefficients.

At very low frequency, the synchrotron radiation spectral index is related to the energy spectrum index by:

$$
\alpha=\frac{\eta-1}{2}
$$

The curves $\alpha(\theta)$ and $\alpha\left(\beta^{u}\right)$ are plotted in Fig. 3. We have limited the computation to $\theta>\theta_{\text {lim }}$ where $\theta_{\text {lim }}$ is given by the condition $r_{g}=u_{0} / u^{d} \geq 1.5\left(u_{0}\right.$ is the speed of the scattering centers at the limit between the precursor and the classical subshock). We have also assumed that $r_{g}$ is given by the parallel case (see Paper 2) and it can be checked on Fig. 2 and 3 of Paper 2 that this assumption does not change the result very much.

\section{Evolution of the cut-off with obliquity}

The cut-off is defined by the balance of energy gain through the acceleration process with the synchrotron losses. We consider here a mean cut-off averaged over space on a scale larger than the synchrotron loss characteristic length. To derive the cut-off frequency, we set:

$$
\lambda_{\text {acc }} \simeq \lambda_{\text {loss }},
$$

where

$$
\lambda_{a c c}=\frac{D}{u^{u}}
$$

and

$$
\lambda_{\text {loss }}=u^{u} \tau_{s y n}=u^{u} \frac{6 \pi m c}{\sigma_{T} \gamma B^{2}}
$$

(Rybicki and Lightman 1979). $\sigma_{T}$ is the Thomson cross section and $D$ the diffusion coefficient. In the case of an oblique shock, this coefficient is given by (Jokipii 1966; Melrose 1980):

$$
D=D_{/ /} \cos ^{2} \beta^{u}+D_{\perp} \sin ^{2} \beta^{u}
$$


with

$$
D_{/ /}=\frac{1}{3} \frac{c^{2}}{\nu_{s}}
$$

and

$$
D_{\perp}=\frac{1}{3} \nu_{s} r_{g r}^{2}=\frac{\nu_{s}}{3} \frac{\left(\gamma^{2}-1\right) m^{2} c^{4}}{e^{2} B^{2}}
$$

where

$$
\nu_{s}=\eta_{t u r b} \frac{\nu_{A}-1}{\nu_{A}\left(\nu_{A}+2\right)} \frac{3 \pi}{2} \frac{c}{l_{0}}\left(\frac{r_{g r}}{l_{0}}\right)^{\nu_{A}-2}
$$

is the pitch angle scattering frequency. $\eta_{t u r b}$ is the degree of magnetic turbulence $\frac{\left\langle(\delta B)^{2}\right\rangle}{\langle B\rangle^{2}}$, $l_{0}$ the long spatial wavelength cut-off of the spectrum, $\nu_{A}$ is the power law of the spectrum of the Alfven waves $\left(\omega^{-\nu_{A}}\right)$ and $r_{g r}=m c^{2} / e B$.

We then obtain a relation between $\beta^{u}$ and $\gamma$ from equations (30) through (36):

$$
\cos ^{2} \beta^{u}=\frac{\frac{18 \pi m}{\sigma_{T} c} \frac{K}{\gamma\left(\gamma^{2}-1\right)^{1-\nu_{A} / 2}} \frac{u^{u 2}}{B^{2}}-\left(\frac{K m c}{e B}\right)^{2}\left(\gamma^{2}-1\right)^{\nu_{A}-1}}{1-\left(\frac{K m c}{e B}\right)^{2}\left(\gamma^{2}-1\right)^{\nu_{A}-1}}
$$

where

$$
K=\eta_{\text {turb }} \frac{\nu_{A}-1}{\nu_{A}\left(\nu_{A}+2\right)} \frac{3 \pi}{2} \frac{c}{l_{0}}\left(\frac{m c^{2}}{l_{0} e B}\right)^{\nu_{A}-2} .
$$

The synchrotron theory provides a relation between the frequency and $\gamma$ with $\nu_{c}=$ $1.210^{6} B \gamma^{2}$ where $B$ is in Gauss. In Fig. 4 is plotted $\beta^{u}\left(\nu_{c}\right)$ given by equation (37).

\section{Discussion}

\subsection{The spectral index}

The evolution of the low frequency radio spectral index $\alpha$ as a function of the obliquity $\beta^{u}$ is represented on Fig. 3a. It shows clearly that the variations of $\alpha$ with $M_{A}$ are roughly constant but are higher at $\beta^{u} \simeq 60-70^{\circ}$. This shows that the magnetic pressure has a relatively weak influence on $\alpha$. Fig. 3a also reveals that the obliquity is not a crucial parameter to determine the spectral index. Fig. 3b is a generalization of the curves presented in Paper 1 and Paper 2. It shows that the variations of $\alpha$ with $M_{A}$ are roughly constant when $\theta$ varies.

The different parameters can be estimated by comparison with observed spectral indices. Unfortunately, the spectrum must be precisely known in order to avoid the uncertainty of 0.5 near the break frequency. The spectral index used here is the index when the synchrotron losses are not significant, it is to say for low energy electrons. Above the energy corresponding to the break frequency, the synchrotron spectrum steepens by 0.5 because of the synchrotron energy losses of the electrons. This break frequency is different for each radio jet and can be between $10^{9}$ and $10^{11} \mathrm{~Hz}$ approximatively. As it is not known 
for all the sources, use of an observed distribution of spectral indices (see for example Bridle and Perley 1984) would probably lead to uncorrect results. We thus prefer to study few sources for which both the break and the cut-off frequencies are known.

\subsection{The cut-off frequency}

The importance of all the parameters intervening in the determination of the cut-off frequency is shown in Fig. 4. We have chosen the most reasonable parameters and considered some variations:

-The index $\nu_{A}$ of the Alfven wave spectrum is in principle either $5 / 3$ (Kolmogorov spectrum) or $3 / 2$ (Kraichnan spectrum). Fig. 4a shows that this parameter is the most important one and that the Kolmogorov spectrum does not allow optical emission except for very high obliquities which are excluded in this paper (the calculations are limited to the quasi-perpendicular case).

-The second important parameter is the turbulence rate which is totally unknown in extragalactic jets. Fritz (1989) find $\eta_{\text {turb }}<3 \quad 10^{-2}$ in 3C 111 and $0235+164$. Roland et al. (1988) estimated that $\eta_{t u r b} \simeq 510^{-4}$ in the hotspot of Cygnus A. We thus choose $10^{-3}$ as a typical value and Fig. $4 \mathrm{~b}$ reveals that only greater turbulence rates can explain optical emission. As only very few extragalactic optical jets exist, we conclude that $\eta_{\text {turb }}$ must be as low as $10^{-3}$ in most cases.

-The calculations developed in Paper 1, in Paper 2 and here assume non relativistic transport equations, i.e. $u^{u}<c / \sqrt{3}=0.58 c$. In addition, Fig. $4 \mathrm{c}$ shows that the influence of the plasma speed on the cut-off frequency is weak for $u^{u}>0.3 c$.

-The influence of the largest scale of the magnetic turbulences is not important. We adopted a typical value of $100 \mathrm{pc}$, corresponding to the knot size in the M87 jet and to the value found by Roland et al. (1988) in the hotspot of Cygnus A.

-The equipartition magnetic field is in general about $10^{-4} \mathrm{G}$ and does probably not differ dramatically from this value. Its influence on the cut-off frequency is thus relatively weak (Fig. 4e).

Regarding to the two first points, it could seem paradoxical that a weak turbulence spectrum of Kraichnan type is a better explaination of the optical cut-off than the strong turbulence spectrum of Kolmogorov type. The reason is that the cut-off frequency is proportional to $\left(l_{0} / r_{g r}\right)^{2\left(2-\nu_{A}\right) /\left(3-\nu_{A}\right)} \eta_{t u r b}^{2 /\left(3-\nu_{A}\right)}$. The first factor is a very large number, very sensitive to the exponent, whereas the second factor, that shows the increase of the cut-off with the turbulence level, is less sensitive.

\subsection{Validity of the theory}

The low level of the MHD turbulence insures the validity of the formulae used for the diffusion coefficients derived from quasilinear theory (Jokipii 1966) and the pitch angle diffusion frequency is much smaller than the Larmor frequency. However the pitch angle frequency cannot be too small because the diffusive acceleration works only if the diffusion length is smaller than the size of the shock region. The largest diffusion length is reached by particles having an energy corresponding to the cut-off frequency (their Lorentz factor is about few $10^{6}$ ). That maximum diffusion length is about $10 \mathrm{pc}$ for $\eta_{\text {turb }}$ equal to $10^{-3}$. 
We note that the isotropization process is always faster than the homogeneization process in non relativistic flow. Indeed the requirement that $u^{u} / \nu_{s}$ be smaller than the diffusion length is equivalent to $u^{u}<<c$.

We considered a quasi-adiabatic shock. Is it consistent with the energy spectral index that turns out to be smaller than 2 just behind the shock? The answer is yes for three reasons. First, after the shock the integrated energy distribution undergoes a change of index due to synchrotron losses leading to the usual increase of the synchrotron index of 0.5. Second, except for particles having an energy close to the cut-off, the diffusion length is much smaller than the characteristic length of synchrotron loss. Third, because of the cut-off, the integrated luminosity is much smaller than the flux of kinetic energy in the jet, by a factor of few percent (see Roland et al. 1988).

\section{Application to the "hot-spot" of the 3C 273 jet}

Instead of deriving general properties for the jets with the uncertainties on the spectral index and the often unknown cut-off frequency, we illustrate the calculations of this paper with one well studied object. The best candidates are certainly the optical jets whose spectrum is also known toward very low radio-frequency in order to determine its spectral index below the break frequency. The cut-off frequency, the equipartition magnetic field and an estimation of the maximum scale of turbulences $\left(l_{0}\right)$ are determined from the observations. For illustration, we consider the "hot-spot" of the jet of 3C 273 whose detailed optical photometry (Fraix-Burnet and Nieto 1988) provides a magnetic field at equipartition of about $10^{-3} \mathrm{G}$ and precise the shape of the cut-off with a cut-off frequency at about $10^{15} \mathrm{~Hz}$. The whole spectrum of this "hot-spot" (see Meisenheimer and Heavens 1986) yields a low-frequency spectral index of 0.5. From the observations (notably Foley and Davis 1985, and Fraix-Burnet et al. 1989b) we estimated the maximum scale length of the turbulence to be about $1 \mathrm{kpc}$.

We are thus able to present the result of Section 3 in a different way, i.e. with two plots: $M_{A}$ vs $\theta$ and $\eta_{\text {turb }}$ vs $u^{u}$. Obliquities of 40,60 and $80^{\circ}$ were choosen in Fig. 5 and the second plot is shown for both Kolmogorov and Kraichnan turbulence spectra. Our calculations have two limitations which are constraints on some parameters: the use of classical transport equations implies $u^{u}<c / \sqrt{3} \simeq 0.6 c$, and because we have considered only quasi-perpendicular shocks we have: $\left(u^{u}+V_{A} \cos \beta^{u}\right) \tan \beta^{u}<c$. With the $\eta_{\text {turb }}\left(u^{u}\right)$ plot, the second condition gives:

$$
\begin{aligned}
& \eta_{\text {turb }}>0.15 \quad \text { for } \quad \nu_{A}=1.7 \\
& \eta_{\text {turb }}>2.510^{-3} \quad \text { for } \quad \nu_{A}=1.5
\end{aligned}
$$

On one hand, the Kolmogorov turbulence spectrum requires quite high degrees of magnetic turbulence, but on the other hand, if such high degrees are to be present, the Kraichnan spectrum would require quite low speed of less than about $0.1 \mathrm{c}$. We tend to believe that the most plausible configuration is a Kraichnan spectrum with a midly relativistic speed $(0.3 \mathrm{c})$ and a low degree of turbulences $\left(10^{-3}-10^{-2}\right)$.

The $M_{A}(\theta)$ plot clearly shows that $M_{A}>5$ whatever the obliquity is. Using $u^{u}<0.6 c$, this yields $V_{A}<0.15$ c, or: 


$$
n>210^{-3} \mathrm{~cm}^{-3}
$$

where $n$ is the density of the thermal plasma. The upper limit on $u^{u}$ provides also an upper limit on $M_{A}$ which can be written as:

$$
5<M_{A} \lesssim 80 n^{+1 / 2}
$$

A lower limit on $\theta$ can be derived from Fig. 5a: $\theta>0.3$.

Whereas it is difficult to go further on in the determination of the parameters of the model for 3C 273 at the present time, it appears that the estimation of the thermal plasma density should bring interesting results. This estimation can be made essentially in two ways: from the X-ray emission of the insterstellar medium with an assumption of some equilibrium between this medium and the jet, and also from internal Faraday depolarization when this effect can be measured. Both method are possible in the jet of M87 (Schreier et al. 1982, Fraix-Burnet et al. 1989a) and we intend to publish in a subsequent paper a thorough study of this jet in the frame of the present model and considering all the data available at all wavelengths.

Any further constraint on the thermal plasma density are lacking in the case of $3 \mathrm{C} 273$, but for illustration we use the value derived by Fraix-Burnet et al. (1989a) in the jet of M87. For $n=210^{-2} \mathrm{~cm}^{-3}$, we derive for a Kraichnan spectrum $\left(\nu_{A}=1.5\right)$ :

$$
\begin{aligned}
0.2 & <u^{u} / c<0.6 \\
5 & <M_{A}<11 \\
0^{\circ} & <\beta^{u}<78^{\circ} \\
0.3 & <\theta<10 \\
310^{-3} & <\eta_{\text {turb }}<410^{-2}
\end{aligned}
$$

\section{Conclusion}

This analysis of oblique shocks, that takes into account magnetic effects in the shock stucture, leads us to emphasize the following concluding points. i) The most sensitive effect of the ordered magnetic field on the shock structure and the resulting energy spectrum is due to the magnetic pressure. ii) The high energy cut-off is so sensitive to the Alfven spectral index $\nu_{A}$ that we can conclude that the MHD turbulence is more likely of Kraichnan rather than Kolmogorov type . iii) The degree $\eta_{t u r b}$ of magnetic turbulence is the other sensitive parameter, however less sensitive than the turbulence spectral index. We found that it is of order $10^{-3}$ for most jets, except for those which radiate an optical synchrotron emission which requires a larger degree $\eta_{t u r b}$ but smaller than $10^{-1}$. Thus, in any case, the MHD turbulence is fairly weak and could not be of Kolmogorov type, which is in agreement with polarization data and the ordered character of the magnetic maps. iv) The theory of diffusive acceleration in oblique shocks works quite well to explain the synchrotron spectrum of extragalactic jets. All the assumptions of the theory turn out to be consistent in the sense that the parameters deduced from the shock theory lead to justify the a priori assumptions. Especially three main assumptions are proposed: most 
of the mass is in a thermal plasma, the flow speed is not relativistic (precisely, below the relativistic sound speed $c / \sqrt{3}$ ), the MHD turbulence that causes the diffusion is weak. We showed that these assumptions are valid and found estimates of these quantities. For the particular exemple of $3 \mathrm{C} 273$, we determined the parameters in rather narrow intervals. And although this source is a powerful quasar, the radiation of its jet is explained with a nonrelativistic flow speed in the "hot spot" region, the relativistic particles being advected by a thermal plasma of few $10^{-3}$ part $/ \mathrm{cm}^{3}$.

\section{Acknowledgements}

We whish to thank an anonymous referee for his quick and very pertinent report. We are also indebted to him for having pointed out an error which in fact originated from Paper 2 . 


\section{References}

Axford, W. I., Leer, E., Skhadron, G. 1977, 15th International Conference on Cosmic Rays, Plovdiv

Begelman, M. C., Kirk, J. G. 1990, Ap. J., 353, 66

Bell, A. R. 1978, M. N. R. A. S., 182, 147

Blandford, R. D., Ostriker, J. P. 1978, Ap. J. (Letters), 221, L29

Bridle, A. H., Perley, R. A. 1984, Ann. Rev. Astr. Ap., 22, 319

Cawthorne, T. V., Cobb, W. K. 1990, Ap. J., 350, 536

Drury, L. O'C. 1983, Rep. Prog. Phys., 46, 973

Foley, A. R., Davis, R. J. 1985, M. N. R. A. S., 216, 679

Fraix-Burnet, D., Le Borgne, J.-F., Nieto, J.-L. 1989a, Astr. Ap., 224, 17

Fraix-Burnet, D., Nieto, J.-L. 1988, Astr. Ap., 198, 87

Fraix-Burnet, D., Nieto, J.-L., Roques, S. 1989b, Astr. Ap., 217, 387

Fritz K.-D. 1989, Ap. J., 347, 692

Jokipii, J. R. 1966, Ap. J., 146, 480

Kirk, J. G., Heavens, A. F. 1989, M. N. R. A. S., 239, 995

Krimsky, G. F. 1977, Sov. Phys. Dokl., 23, 327

Mc Kenzie, J. F., Volk, H. J. 1982, Astr. Ap., 116, 191

Meisenheimer, K., Heavens, A. F. 1986, Nature, 323, 419

Melrose, D. B. 1980, in Plasma Astrophysics, vol. II, Gordon and Breach

Norman, M. L., Smarr, L. L., Winkler, K.-H. A., Smith, M. D. 1982, Astr. Ap., 113, 285

Ostrowski, M. 1988, M. N. R. A. S., 233, 257

Pelletier, G., Roland, J. 1986, Astr. Ap., 163, 9 (Paper 1)

Pelletier, G., Roland, J. 1988, Astr. Ap., 196, 71 (Paper 2)

Roland, J., Pelletier, G., Muxlow T. W. B. 1988, Astr. Ap., 207, 16

Rybicki, G. B., Lightman, A. P. 1979, Radiative Processes in Astrophysics, Wiley, NewYork

Schreier, E. J., Gorenstein, P., Feigelson, E. D. 1982, Ap. J., 261, 42

Skilling, J. 1975, M. N.R. A. S., 172, 557

Völk, ,H.J., Drury, L. O'C., McKenzie, J.F. 1984, Astr. Ap., 130, 19

Webb, G. M. 1983, Astr. Ap., 127, 97 


\section{Figure captions}

Figure 1: Reference frame of the shock front considered in this paper.

Figure 2: Evolution of the compression ratio $r$ as a function of the obliquity $\beta^{u}$ for different values of $\theta$. The curves correspond upward to $M_{A}=4,5,7,10,30, \infty$.

Figure 3: Evolution of the spectral index $\alpha$ with (a) the obliquity $\beta^{u}$ and (b) $\theta$. The curves correspond downward to $M_{A}=4,5,7,10,30, \infty$.

Figure 4: Obliquity $\beta^{u}$ (in degrees) as a function of the cut-off frequency $\nu_{c}(\mathrm{in} \mathrm{Hz})$ for various values of the parameters. The speeds are in unit of $\mathrm{c}$.

Figure 5: For the "hot-spot" of the 3C 273 jet, these curves represent (a) the Alfvenic Mach number $M_{A}$ as a function of $\theta$, and the degree of turbulence $\eta_{t u r b}$ as a function of the flow speed $u^{u}$ for (b) a Kolmogorov spectrum and (c) a Kraichnan spectrum. The speeds are in unit of $c$. 


\section{Addresses}

DIDIER FRAIX-BURNET:

Observatoire Midi-Pyrénées, Observatoire de Toulouse, URA 285, 14 Avenue Edouard Belin, 31400 Toulouse, France.

GUY PELLETIER:

Observatoire de Grenoble, Groupe d'Astrophysique de l'Université de Grenoble 1, URA 708, CERMO, BP 53X, 38041 Grenoble Cedex, France. 